Jap. J. M. Sc. \& Biol., 11, 375-382, 1958

\title{
STUDIES ON STREPTOLYSIN O AND ANTISTREPTOLYSIN O \\ III. A PRACTICAL METHOD FOR THE ANTISTREPTOLYSIN O TITRATION OF HUMAN SERA
}

\author{
HIDEO KUSAMA \\ Department of Bacteriology, National Institute of Health, Tokyo \\ (Received: October 3rd, 1958)
}

In the previous reports (Kusama et al., 1958 a, b), the spectrophotometric determination of the hemolytic activity of streptolysin O (SL) and some fundamental aspects of the streptolysin $\mathrm{O}$ - antistreptolysin $\mathrm{O}$ reaction were presented. The qualitative difference of antistreptolysin $\mathrm{O}$ (ASL) in human sera, as manifested by different slopes of reaction curves and different combining velocities, was considered probably to be due to the avidity difference. A spectrophotometric method for the ASL titration which could minimize this qualitative difference by using a larger amount of SL than employed by other workers was described, and a standard ASL was prepared from a human serum with reference to the Danish standard ASL.

Since the spectrophotometric method is rather laborious and time-consuming, a simple method which could be suited for the routine diagnostic work is considered in this report.

The principle of the method is to compare visually the degree of hemolysis in a number of tubes containing known, varying amounts of the standard ASL with that in a dilution series of the unknown serum, using a constant dose of SL. A dilution of the unknown serum and a known amount of the standard ASL (expressed in units per cc), which would each cause $50 \%$ hemolysis, will be estimated by direct comparison or interpolation. Multiplying the former (the degree of dilution) by the latter (the number of units) will give the units of ASL per cc of the unknown serum.

\section{MATERIALS}

1. Reagents employed: $\mathrm{SL}$, lot 2, rabbit red blood cell (RBC) suspension, buffered saline solution (BS), BS containing $0.1 \%$ bovine serum albumin (Armour fraction V) (AB), and neutralized cysteine solution were prepared as reported previously (Kusama et al., 1958 a, b).

2. Sera examined: T-series (scarlet fever patients' sera) and B-series (Blood Bank donors' sera) were the same as described previously (Kusama et al., 1958 b).

3. Standard ASL: A human serum standardized against the Danish standard ASL (Kusama et al., 1958 b) was used, being properly diluted with BS so that $1.0 \mathrm{cc}$ contained 10 units.

草間秀夫 (国立予防簗生研究所細菌部) 


\section{Methods}

\section{Adjustment of the Test Dose of SL}

As described previously, the SL will be used in such an amount that would cause partial hemolysis mixed with 3.2 units of ASL. This dose will be accurately determined by the spectrophotometric measurement, testing varying amounts of SL against 3.2 units of ASL (Kusama et al., $1958 \mathrm{~b}$ ). However, for the practical purpose, the test dose will be satisfactorily determined as follows :

1. Dilution series for the standard ASL: Several dilution series of the standard ASL were made on $0.05 \log$ unit dilution scale with $\mathrm{BS}$, in $1.0 \mathrm{cc}$-amounts, each series was to receive different SL dose (Table 1).

Table 1. Adjustment of the test dose of the SL, lot 2

\begin{tabular}{|c|c|c|c|c|c|c|c|c|c|c|c|c|c|}
\hline \multicolumn{14}{|c|}{ Degree of hemolysis } \\
\hline \multicolumn{5}{|c|}{ Dose of SL* } & \multicolumn{9}{|c|}{ ASL units per tube } \\
\hline $\begin{array}{c}1: 10 \\
\text { diluted } \\
\text { SL }\end{array}$ & + & $\mathrm{AB}$ & + & $\begin{array}{c}1 / 25 \mathrm{M} \\
\text { cysteine } \\
\text { solution }\end{array}$ & 5.0 & 4.5 & 4.0 & 3.6 & 3.2 & 2.8 & 2.5 & 2.2 & 2.0 \\
\hline $\begin{array}{c}c c \\
0.56\end{array}$ & & $\begin{array}{c}c c \\
0.44\end{array}$ & & $\begin{array}{r}\text { cc } \\
1.0\end{array}$ & 20 & 40 & 100 & 100 & 100 & 100 & 100 & 100 & 100 \\
\hline 0.50 & & 0.50 & & 1.0 & 0 & 20 & 40 & 100 & 100 & 100 & 100 & 100 & 100 \\
\hline 0.45 & & 0.55 & & 1.0 & 0 & 0 & 20 & 40 & 100 & 100 & 100 & 100 & 100 \\
\hline 0.40 & & 0.60 & & $1.0 * *$ & 0 & 0 & 0 & $\operatorname{Tr}$ & 40 & 100 & 100 & 100 & 100 \\
\hline 0.36 & & 0.64 & & 1.0 & 0 & 0 & 0 & 0 & $\operatorname{Tr}$ & 60 & 100 & 100 & 100 \\
\hline 0.32 & & 0.68 & & 1.0 & 0 & 0 & 0 & 0 & 0 & $\operatorname{Tr}$ & 80 & 100 & 100 \\
\hline 0.28 & & 0.72 & & 1.0 & 0 & 0 & 0 & 0 & 0 & 0 & $\operatorname{Tr}$ & 80 & (100) \\
\hline
\end{tabular}

* $0.5 \mathrm{cc}$ of each mixture were added to the ASL dilutions.

** This dose, or $1: 25$ diluted in $A B$ and mixed with an equal volume of $1 / 25 \mathrm{M}$ cysteine solution was selected as the test dose.

2. Dilution series for $S L$ : A proper dilution of the SL, lot 2, was first made in $\mathrm{AB}$ (to be determined by the pilot titration, testing serial 2-fold dilutions of SL against 3.2 units of the standard ASL), and it was further diluted on $0.05 \mathrm{log}$ unit dilution scale with $\mathrm{AB}$. Each dilution then received an equal volume of $1 / 25 \mathrm{M}$ cysteine solution and was kept at room temperature for 10 minutes.

3. Neutralizing and hemolytic reactions: The tubes in each dilution series of the standard ASL then received $0.5 \mathrm{cc}$ of the above activated SL of different dilution. The tubes were kept in a $37^{\circ} \mathrm{C}$ water bath for 1 hour, received $0.5 \mathrm{cc}$ of a $2.5 \% \mathrm{RBC}$ suspension each, and placed again in the bath for 1 hour. A part of the protocols on such tests is given in Table 1, where a $1: 25$ dilution of the SL, lot 2 , mixed with an equal volume of $1 / 25 \mathrm{M}$ crysteine solution will be suitable for the test dose. The details of the test and reading the results will be given in the next chapter. 


\section{Visual Determination of $A S L$}

1. Dilution series for the standard ASL: Varying amounts of BS were taken in 5 tubes, as shown in Table 2, and the standard ASL (10 units per cc) was added as indicated, in $1.0 \mathrm{cc}$-volumes, so that the serum was diluted on $0.05 \mathrm{log}$ unit dilution scale.

2. Dilution series for the test serum: As shown in Table 2, Dilution I (1:10) and II $(1: 100)$ were first prepared with BS. The indicated amounts of BS were distributed to Tube No. 1 to 11 . Undiluted serum, Dilution I and II were then added as indicated, to make the total volume of $1.0 \mathrm{cc}$. Thus, the serum was diluted on $0.2 \mathrm{log}$ unit dilution scale. As the dilution started at a rather low level, a strongly hemolyzed serum could not be tested, especially when it had a low titer.

Table 2. Dilution series for the standard and test serum in the visual method

Standard ASL

\begin{tabular}{cccccc}
\hline Tube No. & 1 & 2 & 3 & 4 & 5 \\
\hline $\begin{array}{l}\text { Standard ASL } \\
(10 \text { units/cc), cc }\end{array}$ & 0.40 & 0.36 & 0.32 & 0.28 & 0.25 \\
\hline BS, cc & 0.60 & 0.64 & 0.68 & 0.72 & 0.75 \\
\hline ASL units per tube & 4.0 & 3.6 & 3.2 & 2.8 & 2.5 \\
\hline
\end{tabular}

Test serum

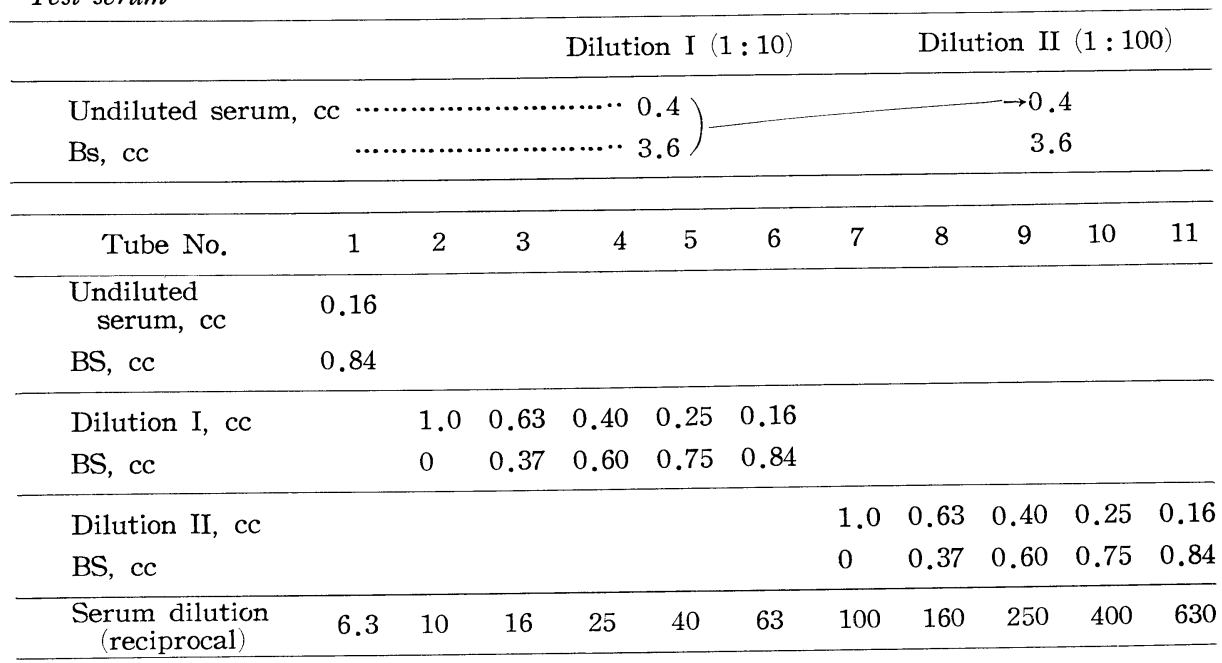

3. Control tubes: A tube containing $1.5 \mathrm{cc}$ of $\mathrm{BS}$ and a tube containing $1.0 \mathrm{cc}$ of BS were always included for the RBC control and SL control, respectively.

4. Neutralizing and hemolytic reactions: All the tubes, except the RBC control, then received $0.5 \mathrm{cc}$ of the activated $\mathrm{SL}^{*}$, lot 2 , each, the dosage of which

* Diluted in $\mathrm{AB}$, mixed with an equal volume of $1 / 25 \mathrm{M}$ cysteine solution, and kept at room temperature for 10 minutes before use. 
had been adjusted, shaken thoroughly and placed in a $37^{\circ} \mathrm{C}$ water bath for 1 hour. Then, $0.5 \mathrm{cc}$ of a $2.5 \% \mathrm{RBC}$ suspension was distributed to each tube. ${ }^{*}$ The tubes were placed again in the bath for additional 1 hour, being shaken at 15 minute intervals, and then centrifuged at $1,500 \mathrm{rpm}$ for 1 minute.

5. Visual reading of the degree of hemolysis: The degree of hemolysis was registered as $0, \operatorname{Tr}, 20,40,60,80,(100)$ and $100 \%$ of the total hemolysis, by visual comparison with a series of tubes showing 20,40,60 and $80 \%$ hemolysis, which were made by diluting a pooled sample from several tubes showing complete hemolysis. The amount of the $\mathrm{RBC}$ sediment was also taken into account in evaluating the degree of hemolysis.

6. Calculation of the ASL titer: In determing titer, a table (Table 3) was made on the same principle as in Ipsen's table (Ipsen, 1944). Ipsen compared a tube in the test serum series showing partial hemolysis with the standard series, and found the tube in the latter series showing the same degree of hemolysis, or 2 tubes between which the degree of hemolysis of the test serum sample lay. However, the author compared the dilution of the test serum and the standard

Table 3. Calculation of the ASL titer

\begin{tabular}{|c|c|c|c|c|c|c|c|c|c|c|c|c|c|c|c|c|c|c|c|c|c|}
\hline \multirow{2}{*}{\multicolumn{3}{|c|}{$\begin{array}{c}\text { Dilution } \\
\text { (reciprocal) }\end{array}$}} & \multicolumn{18}{|c|}{ Test serum series } & \multirow[b]{2}{*}{630} \\
\hline & & & 6.3 & 10 & 16 & & 25 & & 40 & & 63 & & 100 & & 160 & & 250 & & 400 & & \\
\hline \multirow{6}{*}{ 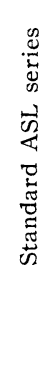 } & Units & Tube No. & 1 & 2 & 3 & & 4 & & 5 & & 6 & & 7 & & 8 & & 9 & & 10 & & 11 \\
\hline & 4.0 & 1 & 2532 & 405 & 63 & & 100 & 1251 & 1602 & 200 & 250 & 320 & 400 & 500 & 6308 & 800 & 1000 & 1250 & 16002 & 20002 & \\
\hline & 3.6 & 2 & 22 & 364 & 56 & & 90 & 1101 & 140 & $\begin{array}{c}180 \\
!\end{array}$ & 2202 & 280 & 360 & 450 & 560 & 500 & 900 & 1100 & 14001 & 1800 & 2200 \\
\hline & 3.2 & 3 & 2025 & 324 & 50 & 3 & 80 & 100 & 125 & 160 & 200 & $\frac{1}{250}$ & 320 & 400 & 500 & 630 & 800 & 1000 & 1250 & 1600 & 20 \\
\hline & 2.8 & 4 & 18 & 283 & 645 & 56 & 70 & 901 & 110 & 140 & 180 & $\begin{array}{c}1 \\
220 \\
1\end{array}$ & 280 & 360 & 450 & $\begin{array}{c}1 \\
560 \\
1\end{array}$ & 700 & 900 & $11 \mathrm{co}$ & 1400 & 1800 \\
\hline & 2.5 & 5 & 162 & 253 & 40 & & 63 & & 100 & $\begin{array}{c}125 \\
!\end{array}$ & 160 & 200 & 250 & 320 & 400 & $5 C 0$ & 630 & 800 & $1 C 00$ & 1250 & 1600 \\
\hline
\end{tabular}

serum which would produce $50 \%$ hemolysis (really observed or interpolated), according to Packalén and Bergqvist's technique (1947) applied to the antistaphylolysin determination. Although they regarded 40-60\% hemolysis as 50\%, 0-20\% hemolysis as $0 \%$ and $80-100 \%$ hemolysis as $100 \%$, partial hemolysis between 20 and $80 \%$ was regarded as $50 \%$ in the present method. The reason for this will be explained by an example of the reaction curve of the standard ASL determined by the spectrophotometric method (Fig. 1).

If the reaction of a tube occurs at (1) with $20 \%$ hemolysis, the next tube's reaction will be $100 \%$ hemolysis, occurring at (2). If $0-20 \%$ hemolysis is regarded as $0 \%$, a $50 \%$ point would be interpolated as $(3)$ which is intermediate between (1) and (2), and the point (3) might differ by as much as $a$ from a $50 \%$ point

* Most of other methods employ a $5 \% \mathrm{RBC}$ suspension. But, the use of a $2.5 \% \mathrm{RBC}$ suspension facilitated the visual comparison of the supernatants, especially for $60-80 \%$ hemolysis. 
which could be obtained by the closer study. On the other hand, if $20 \%$ is regarded as $50 \%$, the end point would differ by $b$ from the point in the closer study. It is the case with $80 \%$ hemolysis. Investigations on reaction curves of other sera also revealed the same situation, owing to steepened slopes by the use of a large test dose of SL. The distance $b$ varies as the shape of the curve changes, but is within $0.05(\mathrm{log})$ for most of sera, and does not seem to exceed 0.1 for a few sera with flat curves.

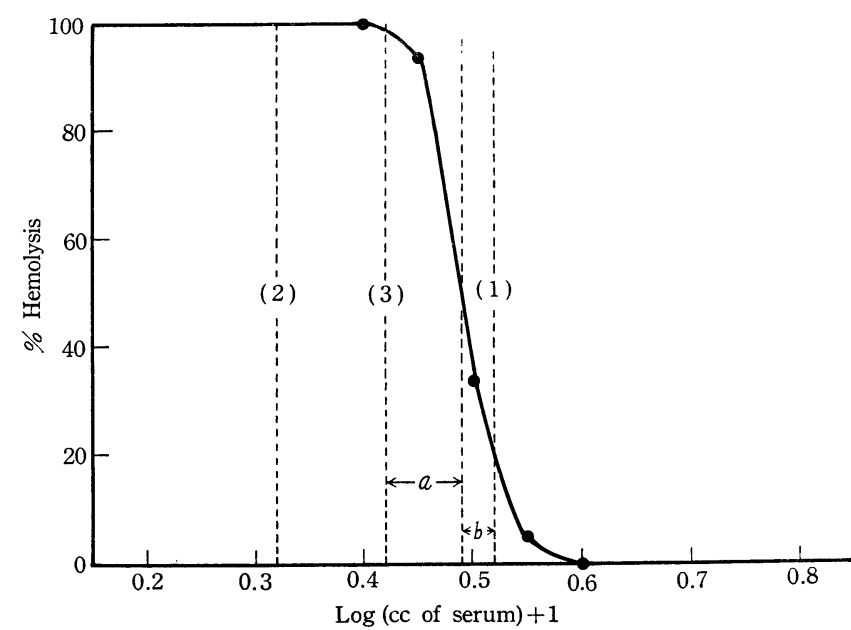

Fig. 1. Reaction curve of the standard ASL (10 units/cc) measured by the spectrophotometric method with $80 \mathrm{H}$.U. of the SL, lot 2. The serum was diluted on $0.05 \mathrm{log}$ unit dilution scale, whereas $0.1 \mathrm{log}$ unit dilution scale was used in the visual method. And, the estimation of a $50 \%$ point in the visual method is illustrated (See text).

In reading Table 3, a horizontal row corresponding to a tube in the standard series, showing $20-80 \%$ hemolysis, was first selected, usually being Tube No. 3 containing 3.2 units of ASL.* The ASL titer was then directly read as the figure at the intersection of the vertical column corresponding to a tube showing $20-80 \%$ hemolysis and the above horizontal row. As a rule, only one tube in the test serum series showed partial hemolysis, or else there were tubes with only total and no hemolysis. In the latter case where no partial hemolysis occurred, a $50 \%$ point was interpolated which was intermediate between the last tube with 0 or $\mathrm{Tr}$ hemolysis and the next following with (100) or $100 \%$ hemolysis. Thus, the titer was read on $0.1 \mathrm{log}$ unit scale.

If partial hemolysis between 20 and $80 \%$ occurs in more than 2 tubes indicating an extremely flat curve, the titer can not be determined. Kalbak (1947) stated that flat reaction curves were observed in testing sera from icteric or

* In more than 30 routine assays thus far conducted, the degree of hemolysis in Tube No. 3 was very constant, always lying between $20-80 \%$ hemolysis, mostly $40-60 \%$, and never showed 0 , Tr or $(100), 100 \%$ hemolysis. Tube Nos. 2 and 4 constantly showed 0 or $\operatorname{Tr}$ and $(100)$ or $100 \%$ hemolysis, respectively. 
nephrotic patients, possibly owing to the non-specific inhibition of hemolysis by increased quantities of cholesterol or other abnormal serum lipids. Although enough materials have not been available for this study, some fragmental data supported his statement. Moreover, such inhibition of hemolysis seemed not to follow the low of multiple proportions which was observed in the true SL-ASL reaction.

\section{Spectrophotometric Determination of $A S L$}

In order to evaluate the accuracy of the visual method, the spectrophotometric method reported in the preceding paper (Kusama et al., $1858 \mathrm{~b}$ ) was simultaneously carried out, using $1.0 \mathrm{cc}$ of serum dilutions on $0.05 \mathrm{log}$ unit dilution scale for both the standard and test serum, $2.0 \mathrm{cc}$ of the activated SL, lot 2, and $1.0 \mathrm{cc}$ of a $2.5 \%$ RBC suspension for each tube. The test dose of the SL was $80 \mathrm{H} . \mathrm{U}$. which caused $50 \%$ hemolysis with 3.2 units of ASL, as reported previously.

Instead of transforming per cent hemolysis into probit, a sigmoid curve was drawn by direct plotting of per cent hemolysis against log serum ( $c f$. Fig. 1), and the serum dose corresponding to $50 \%$ hemolysis was interpolated $\left(D_{50}\right)$. Thus, antilog of $\left(\log D_{50}\right.$ of the standard $-\log D_{50}$ of the test serum $) \times 10$ was an estimate of the ASL units per cc of the test serum, since the standard ASL contained 10 units per cc.

\section{RESULTS}

Among the scarlet fever patients' sera ( $\mathrm{T}$-series) and blood donors' sera (Bseries), 2 sera (T-17-4 and T-28-4) belonging to the group of the steepest reaction

Table 4. Tests for accuracy of the visual (A) and spectrophotometric (B) methods

\begin{tabular}{|c|c|c|c|c|c|c|c|c|c|c|c|c|}
\hline \multirow{2}{*}{$\begin{array}{c}\text { Serum } \\
\text { Method }\end{array}$} & \multicolumn{2}{|c|}{$\mathrm{T}-17-4$} & \multicolumn{2}{|c|}{ T-28-4 } & \multicolumn{2}{|c|}{$\mathrm{T}-29-4$} & \multicolumn{2}{|c|}{$\mathrm{T}-30-4$} & \multicolumn{2}{|c|}{ B-122 } & \multicolumn{2}{|c|}{ B-124 } \\
\hline & A & B & A & B & A & B & $\mathrm{A}$ & B & A & B & $\mathrm{A}$ & B \\
\hline \multirow{10}{*}{$\mathrm{ASL} / \mathrm{cc}$} & 630 & 600 & 1000 & 900 & 250 & 270 & 160 & 125 & 63 & 59 & 80 & 73 \\
\hline & 630 & 600 & 1000 & 910 & 320 & 310 & 160 & 130 & 63 & 60 & 80 & 79 \\
\hline & 630 & 630 & 1000 & 980 & 320 & 300 & 160 & 135 & 63 & 58 & 80 & 73 \\
\hline & 630 & 620 & 1000 & 980 & 320 & 270 & 160 & 125 & 63 & 60 & 80 & 79 \\
\hline & 630 & 630 & 1000 & 960 & 320 & 280 & 160 & 120 & 63 & 59 & 80 & 80 \\
\hline & 630 & 630 & 1000 & 960 & 320 & 260 & 160 & 120 & 63 & 62 & 80 & 75 \\
\hline & 630 & 640 & 1000 & 960 & 320 & 290 & 160 & 135 & 63 & 60 & 80 & 73 \\
\hline & 630 & 620 & 1000 & 940 & 320 & 290 & 160 & 130 & 63 & 63 & 80 & 73 \\
\hline & 630 & 610 & 1000 & 940 & 320 & 280 & 160 & 140 & 63 & 62 & 80 & 76 \\
\hline & 630 & 610 & 1000 & 920 & 320 & 300 & 160 & 150 & 63 & 58 & 80 & 73 \\
\hline Mean & 630 & 620 & 1000 & 945 & 310 & 285 & 160 & 130 & 63 & 60 & 80 & 75 \\
\hline S. D. & & 14 & & 27 & & 16 & & 9 & & 1.7 & & 3.0 \\
\hline $\begin{array}{l}\text { S. D./Mean } \\
\times 100\end{array}$ & & 2.3 & & 2.9 & & 5.6 & & 6.9 & & 2.8 & & 4.0 \\
\hline
\end{tabular}


curve, 2 sera (T-29-4 and T-30-4) belonging to the middle group and 2 sera (B122 and B-124) belonging to the flat group were selected to determine the error of measurement in both the visual and spectrophotometric methods. These sera widely differing in the potency were assayed on 10 different days, using the same $\mathrm{RBC}$ suspension for both the methods on each day.

As summarized in Table 4, the spectrophotometric method gave small day-today variations of the calculated titer, and the coefficient of varians varied from 2 to 7 per cent, which did not exactly reflect the steepness of the curve, because T-29-4 and T-30-4 had rather large coefficients in spite of their middle values of the slope, and the flat group sera showed smaller coefficients than the middle group sera.

The repetition of the visual method gave the identical results to each serum, except once to T-29-4. The variations of the day-to-day measurements by this method seems to have little or no effect on the calculated titer, although it will have greater error than the spectrophotometric method. It is due to a certain extent of approximation involved in the visual method, which reads the titer on $0.1 \mathrm{log}$ unit scale (with about $25 \%$ spacing). The table also shows that the difference of the titers given by the two methods are always less than $0.1(\log )$.

\section{Discussion}

It has been the customary practice in the ASL titration of human sera to keep the SL-serum mixtures for 15 minutes at $37^{\circ} \mathrm{C}$ and 45 minutes at $37^{\circ} \mathrm{C}$ after the addition of RBC suspension, since Todd first reported (Todd, 1932).

Hodge and Swift (1933) used the largest quantity of SL which caused no hemolysis in the presence of 1 unit of ASL as the test dose ( 1 combining unit). The end-point of the test was, therefore, the complete inhibition of hemolysis. This method was followed by other workers (Coburn and Pauli, 1935 ; Rantz and Randall, 1945). On the other hand, Scandinavian workers (Kalbak, 1947; Ipsen, 1944) employed the dose of SL which gave partial hemolysis mixed with 0.5 units of ASL and no hemolysis with 0.63 units.

The studies on the neutralization reaction between SL and ASL reported in the preceding paper (Kusama et al., $1958 \mathrm{~b}$ ) disclosed the qualitative difference of ASL in human sera, which seemed to be of profound importance in defining the conditions of the ASL measurement.

The problems are:

1. The neutralization reaction of the test serum should be compared with that of the standard ASL under the conditions where the neutralization of both sera has come or approached closely to an end, unless the combining velocities of both sera are the same. These conditions will be attained by increasing the SL test dose.

2. The difference in the slope and combining velocity between the test and standard serum can also be greatly lessened by raising up the test dose.

3. In routine titration work, where a large number of serum samples are tested simultaneously, the error due to the time required to the distribution of SL or RBC suspension to tubes can not be neglected, if the SL-ASL combination is still in progress. 
The conditions of the present visual method, set up by considering these factors, greatly differ from those used by most of previous workers; the dose of SL is that causes partial hemolysis with 3.2 units of ASL, the combining and hemolysis times are each 1 hour at $37^{\circ} \mathrm{C}$ and $\mathrm{RBC}$ concentration is $2.5 \%$. The conditions used by Halbert et al. (1955) seems somewhat similar to those of the author. They used 30 hemolytic units (H.U.)/0.4 cc of SL which showed $50 \%$ hemolysis in the presence of $0.4 \mathrm{cc}$ of $1: 54$ to $1: 56$ diluted standard ASL (180 units per cc), and 30 minutes at $37^{\circ} \mathrm{C}$ for the SL-ASL combination and 1 hour at $37^{\circ} \mathrm{C}$ for hemolysis, although their method of defining H.U. was differed from that used in the present series of experiments.

Surprising reproducibility of the visual method will be due to the solution of the above problems and the very sharp end-point given by the steepened reaction curve. In general, $0.1(\mathrm{log})$ difference of the titer in repeated measurements may rarely occur, but $0.2(\mathrm{log})$ difference will not occur. Application of this technique to the examination of human sera from various sources are now in progress.

\section{SUMMARY}

1. A practical visual method for the ASL titration of human sera was presented, which intended to minimize the effect of the qualitative difference of ASL in human sera on the results.

2. The accuracy of the measurements by this method and by the spectrophotometric method was examined, and high reproducibility of the visual method suggests that this technique can be satisfactorily applied to the routine diagnostic work.

\section{REFERENCES}

CoBuRn, A. F. \& PAuli, R. H. (1935): Studies on the immune response of the rheumatic subject and its relationship to activity of the rheumatic process. I. The determination of antistreptolysin titer. J. Exper. Med., 62, 129-136.

HALBERT, S. P., Swick, L. \& SonN, C. (1955): The use of precipitin analysis in agar for the study of human streptococcal infections. I. Oudin technic. J. Exper. Med., 101, 539-556.

HODGE, B. E. \& SWIFT, H. F. (1933): Varying hemolytic and constant combining capacity of streptolysins ; Influence of testing for antistreptolysins. J. Exper. Med., 58, 277-287.

IPSEN, J. (1944): A standard for antistreptolysin $O$ of human serum, and its practical application. Acta path. et microbiol. scandinav., 21, 203-213.

KALBAK, K. (1947): The antistreptolysin reaction (A. S. R.). I : Technic. Copenhagen.

Kusama H., OHAShI, M., Shimazaki, H. \& FukUmi, H. (1958 a): Studies on streptolysin $\mathrm{O}$ and antistreptolysin $\mathrm{O}$. I. Spectrophotometric determination of the hemolytic acitivity of streptolysin $\mathrm{O}$ by the fifty per cent end-point titration. Jap. J. M. Sc. \& Biol., 11, 347-357.

Kusama, H., Ohashi, M., Shimazaki, H. \& Fukumi, H. (1958 b): Studies on streptolysin $\mathrm{O}$ and antistreptolysin $\mathrm{O}$. II. Neutralization reaction of streptolysin $\mathrm{O}$ by antistreptolysin O. Jap. J. M. Sc. \& Biol., 11, 359-373.

PACKALÉN, T. \& BERGQVist, S. (1947): Staphylococci in throat and nose and antistaphylolysin titre. Acta med. scandinav., 127, 292-312.

RANTZ, L. A. \& RANDALl, E. (1945): A modification of the technique for determination of antistreptolysin titer. Proc. Soc. Exper. Biol. \& Med., 59, 22-25.

TodD, E. W. (1932): Antigenic streptococcal hemolysin. J. Exper. Med., 55, 267-280. 